[0212-7199 (2003) 20: 11; pp 569-574] ANALES DE MEDICINA INTERNA Copyright @ 2003 ARAN EDICIONES, S.L

AN. MED INTERNA (Madrid) Vol. 20, N. ${ }^{\circ} 11$, pp. 569-574, 2003

\title{
Endocarditis infecciosa en el anciano
}

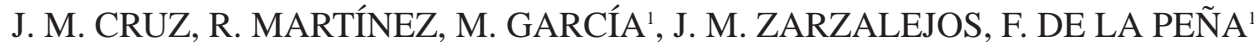 \\ Servicios de Medicina Interna y ${ }^{1}$ Cardiología. Hospital Cristal-Piñor. Ourense
}

INFECTIVE ENDOCARDITIS IN THE ELDERLY

\section{RESUMEN}

Fundamento: La influencia de la edad sobre la presentación clínica y el pronóstico de la endocarditis infecciosa (EI) es desconocida. El objetivo del estudio fue analizar las características epidemiológicas, clínicas y bacteriológicas de la EI en pacientes ancianos y compararlas con las de adultos más jóvenes.

Pacientes y método: Estudio retrospectivo de todos los casos de El en pacientes no usuarios de drogas por vía parenteral diagnosticados en nuestro hospital durante el periodo de 1990 a 2000. Se utilizaron los criterios de Duke para comparar las características de 46 episodios de EI definitiva en pacientes ancianos ( $>65$ años) y de 46 episodios en adultos jóvenes (20-64 años).

Resultados: No encontramos diferencias significativas entre los dos grupos con respecto al retraso en el diagnóstico, a la posible fuente de infección, frecuencia de hemocultívos positivos y tipo de microorganismos, presentación clínica y evolución de la EI. Los ancianos tienen más a menudo un factor de riesgo predisponente (lesiones valvulares degenerativas, válvulas protésicas y marcapasos), que disminuye la sensibilidad de la ecocardiografía transtorácica hasta el 46,5\% comparada con el $64,4 \%$ en los enfermos más jóvenes. La ecocardiografía transesofágica incrementó el diagnóstico de EI en un $37,2 \%$ en los ancianos. Los pacientes de ambos grupos fueron operados con igual frecuencia (el $36,9 \%$ de ancianos y el $39,1 \%$ de los adultos jóvenes), y la tasa de mortalidad no fue significativamente mayor en los ancianos $(19,5 \%)$ que en los pacientes adultos jóvenes $(10,8 \%)$.

Conclusiones: La edad en si misma no es un factor de peor pronóstico, y no debería utilizarse como criterio fundamental para denegar un tratamiento temprano y agresivo a los pacientes con EI.

PALABRAS CLAVE: Endocarditis infecciosa. Anciano. Características clínicas. Pronóstico.

\begin{abstract}
Background: The influence of age on the clinical presentation and on the prognosis of infective endocarditis (IE) is unclear. Our aim was to analyse the epidemiologic, clinical and bacteriological characteristics of $I E$ in the elderly compared with younger adult patients.

Patients and method: A retrospective study of all patients with IE non intravenous drug users diagnosed in our hospital during the period from 1990 to 2000. We used the Duke criteria to compare the characteristics of 46 episodes of definitive IE in elderly patients ( $>65$ years old ) and of 46 episodes in younger adult patients (20-64 years of age ).

Results: No significant differences were observed among the two groups with respect to the delay in diagnosed, possible source of infection, the frecuency of positive blood cultures and the type of infective organism, clinical presentation and evolution of the IE. Elderly patients more often had risk factors predisposing (degenerative valvular disease, prosthetic valve and pacemaker), which decreased the sensitivity of transtoracic echocardiography to $46.5 \%$ compared with $64.4 \%$ in the younger patients. Transesophageal echocardiography improved the diagnostic of IE in the $37.2 \%$ in elderly patients. The patients in the both groups underwent surgical therapy with similar frecuency $(36.9 \%$ in the elderly and the $39.1 \%$ in the younger adult patient) and the mortality rate not was significantly higher in the elderly $(19.5 \%)$ than in the younger adult patients $(10.8 \%)$.

Conclusions: The age itself is not a poor prognostic, and should not be used prejudicially in denying a early and aggressive treatment of the patients with IE.
\end{abstract}

KEY WORDS: Infective endocarditis. Elderly. Clinical features. Prognosis.

Cruz JM, Martínez, R, García M, Zarzalejos JM, de la Peña F. Endocarditis infecciosa en el anciano. An Med Interna (Madrid) 2003; 20: 569574

\section{INTRODUCCIÓN}

La epidemiología de la endocarditis infecciosa (EI) ha cambiado en las últimas décadas, y numerosas publicaciones han descrito un incremento de la incidencia de endocarditis en pacientes ancianos (1-3). Esta tendencia podría continuar por el envejecimiento global de la población y el consiguiente aumento de la esperanza de vida, que en nuestro país es de las más altas dentro de los países desarrollados (4). Además, los pacientes de edad avanzada por sus especiales características: comorbilidad, fragilidad cutánea, inmunosenescencia, ingresos hospitalarios frecuentes, aumento de valvulopatías dege-

Trabajo aceptado: 20 de mayo de 2003

Correspondencia: José Manuel Cruz Vicente. C/ Manuel González Hoyos, 3-6º A. 39012 Santander. 
nerativas y la utilización creciente de material protésico intracardíaco, constituyen un grupo de población predispuesto al desarrollo de este proceso infeccioso $(5,6)$. Sin embargo, existen relativamente pocos datos en la bibliografía y en el momento actual hay discrepancias sobre la influencia de la edad en la presentación y curso clínico de la EI. Mientras en algunos estudios se ha sugerido que los pacientes ancianos tienen una mayor incidencia de manifestaciones atípicas, lo que podría contribuir en este colectivo a un retraso diagnóstico y a una alta tasa de complicaciones $(7,8)$. Otras series clínicas más recientes (9-11), que utilizan los criterios de Duke (12) para la selección de los pacientes, concluyen que las características y evolución de la EI es similar para los ancianos y los pacientes adultos jóvenes. Por ello, el objetivo de este trabajo ha sido estudiar las características clínicas y pronósticas de la EI en el anciano y analizar si existían diferencias con un grupo de pacientes de menor edad.

\section{PACIENTES Y MÉTODOS}

Presentamos un estudio retrospectivo de todos los casos de EI en pacientes no usuarios de drogas por via parenteral diagnosticados en un solo hospital durante un período de 11 años (entre 1990 y 2000, ambos inclusive). Nuestro centro funciona como un hospital terciario de referencia que atiende a una población aproximada de 240.000 habitantes de Orense y su provincia. El hospital no dispone de cirugía cardiaca de forma que en caso de indicación quirúrgica el paciente debía ser trasladado a otros centros, y se recogía información detallada de los hallazgos quirúrgicos y el resultado final.

Se aceptaron para evaluación aquellos pacientes que fueron diagnosticados de EI definitiva según los criterios de Duke (12), y se incluyeron en dos grupos dependiendo de la edad: ancianos ( $\geq 65$ años de edad) y pacientes adultos jóvenes (20-64 años).

Se cumplimentó un protocolo de recogida de datos a partir de la revisión de las historias clínicas de cada uno de los pacientes, que incluía las siguientes variables: edad, sexo, enfermedad cardiaca predisponente, comorbilidad (diabetes mellitus, cardiopatía isquémica, etilismo, enfermedad hepática o cáncer), localización topográfica de la infección y tipo de válvula afectada (nativa o protésica), microorganismo aislado en dos o más hemocultivos obtenidos por venopunciones separadas o estudio serológico, presumible puerta de entrada, tipo de endocarditis, hallazgos ecocardiográficos, demora entre el inicio de los síntomas y/o signos hasta el ingreso del paciente, presencia de fiebre $\left(\geq 38^{\circ} \mathrm{C}\right.$ registrada en dos o más ocasiones durante el ingreso), manifestaciones cardiacas y extracardiacas, necesidad de cirugía y mortalidad durante la hospitalización. Se consideró endocarditis protésica precoz la que sucedió en el primer año tras la sustitución valvular, y protésica tardía la que sucedió después de este periodo. A todos los pacientes se les realizó un ecocardiograma transtorácico y/o transesofágico a criterio clínico. Se utilizaron los criterios ecocardiográficos de EI propuestos por Durack y cols. (12): vegetación, absceso o nueva dehiscencia de una válvula protésica.

Análisis estadístico. Los resultados de las variables cualitativas se expresaron como frecuencias o porcentajes, y las cuantitativas fueron descritas como media aritmética (desviación estandar). Para la comparación de las variables cualitativas entre los dos grupos, se ha utilizado el test de la $\chi^{2}$ o la prueba exacta de Fisher cuando fue necesario, y para las cuantitativas se ha empleado la prueba de la t de Student. Se consideró que existía significación estadística cuando la $\mathrm{p}$ fue menor de 0,05 .

\section{RESULTADOS}

\section{CARACTERÍSTICAS DE LOS PACIENTES}

En el periodo de estudio un total de 92 pacientes fueron identificados para su análisis; 46 en el grupo de ancianos, 34 hombres (74\%) y 12 mujeres (26\%), con una edad media (DE ) de $72,1(5,1)$ años, y 46 pacientes entre 20 y 64 años, de los que 40 eran varones (86\%) y 6 mujeres $(13 \%)$, con una edad media (DE) de 49,1 $(12,8)$ años.

En la tabla I se expone la enfermedad cardiaca predisponente, la localización de la infección, situaciones de comorbilidad y la presumible puerta de entrada de la EI en ambos grupos. Una cardiopatía predisponente ha sido encontrada con mayor frecuencia en los casos de pacientes de edad avanzada $(67,3 \%)$ comparado con el grupo de adultos jóvenes $(60,8 \%)$. La endocarditis sobre material protésico intracardiaco (válvulas protésicas o marcapasos) fue también más común en los ancianos que en el grupo de enfermos más jóvenes $(30,4 \%$ frente al $21,7 \%$, respectivamente), aunque sin diferencias significativas. En los 20 pacientes con EI sobre válvula protésica, predominó la EI protésica tardía y sólo 3 pacientes ancianos y 2 adultos jóvenes padecieron una EI protésica precoz. El tipo de afectación valvular resultó similar entre los dos grupos.

La incidencia de diabetes, cardiopatía isquémica y cáncer fue más alta entre los pacientes de mayor edad que entre los adultos jóvenes, en especial, la existencia de neoplasias $(17,3 \%$ frente al $0 \% ; p=0,005)$. La incidencia de otras situaciones de comorbilidad no fue significativamente distinta en los ancianos comparada con la del grupo de adultos jóvenes.

Se encontró una presumible puerta de entrada del microorganismo causal de la enfermedad en 22 pacientes $(47 \%)$ ancianos y en 20 casos $(43,4 \%)$ del grupo de enfermos más jóvenes. La fuente de infección urológica y el antecedente de manipulación dental se hallaron más a menudo entre los ancianos y el grupo de población de menor edad ( 8,6 frente al $2,1 \%$; y el 8,6 frente al $4,3 \%$, respectivamente). Sin embargo, al igual que en otras fuentes de bacteriemia no existieron diferencias relevantes entre ambos grupos.

\section{MICROBIOLOGÍA}

Globalmente, no fue significativa la diferencia en la distribución de los microorganismos aislados entre los dos grupos (Tabla II). Estreptococos y estafilococos fueron los microorganismos aislados con mayor frecuencia. Así, el 35,8\% de todos los episodios de endocarditis fueron causados por estreptococos y el $31,5 \%$ por estafilococos. Sin embargo, específicamente, la incidencia de infección por estreptococos del grupo viridans ( $8,6 \%$ en los ancianos frente al $23,8 \%$ en los jóvenes; $p=0,049$ ) fue diferente entre ambos grupos.

Los hemocultívos resultaron positivos en el $84 \%$ de los casos en la población anciana y en el $84,8 \%$ en el grupo de menor edad. En tres casos que tenían hemocultivos negativos el diagnóstico se realizó por estudio microbiológico de la válvula. 


\section{TABLA I}

CARDIOPATÍA PREDISPONENTE, LOCALIZACIÓN COMORBILIDAD Y PUERTA DE ENTRADA EN 92 EPISODIOS DE ENDOCARDITIS INFECCIOSA EN PACIENTES ANCIANOS Y ADULTOS JÓVENES

\begin{tabular}{|c|c|c|}
\hline & \multicolumn{2}{|c|}{$\mathrm{N}^{\circ}$ de episodios } \\
\hline & $\begin{array}{l}\text { Ancianos } \\
(\mathrm{n}=46)\end{array}$ & $\begin{array}{l}\text { Adultos jóvenes } \\
\quad(n=46)\end{array}$ \\
\hline \multicolumn{3}{|l|}{ Cardiopatía predisponente } \\
\hline Dispositivos protésicos & $14(30,4)^{a}$ & $10(21,7)^{b}$ \\
\hline No cardiopatía conocida & $15(32,6)$ & $18(39,1)$ \\
\hline $\begin{array}{l}\text { Enfermedad de válvula } \\
\text { nativa }\end{array}$ & $17\left(36^{\prime} 9\right)$ & $18(39,1)$ \\
\hline Enfermedad mitral & 6 & 7 \\
\hline Enfermedad aórtica & 4 & 6 \\
\hline Valvulopatía mitroaórtica & 3 & 2 \\
\hline Cardiopatía congénita & 4 & 5 \\
\hline \multicolumn{3}{|l|}{ Localización } \\
\hline Mitral & $14(30,4)$ & $17\left(36^{\prime} 9\right)$ \\
\hline Aórtica & $25(54,3)$ & $22(47)$ \\
\hline Tricúspide & $4(8,6)^{c}$ & $1(2,1)$ \\
\hline Otras & $3(6,5)$ & $6(13)$ \\
\hline \multicolumn{3}{|l|}{ Comorbilidad } \\
\hline Diabetes mellitus & $6(13)$ & $4(8,6)$ \\
\hline Cardiopatía isquémica & $5(10,8)$ & $1(2,1)$ \\
\hline Insuficiencia renal crónica & $1(2,1)$ & $4(8,6)$ \\
\hline Cáncer & $8(17,3)^{d^{*}}$ & 0 \\
\hline Etilismo & $3(6,5)$ & $7(15,2)$ \\
\hline \multicolumn{3}{|l|}{ Puerta de entrada } \\
\hline Manipulación dental & $2(4,3)$ & $4(8,6)$ \\
\hline $\begin{array}{l}\text { Procedimientos vasculares } \\
\text { invasivos / catéter central } \\
\text { / hemodiálisis }\end{array}$ & $4(8,6)$ & $5(10,8)$ \\
\hline Urológica & 4 & $1(2,1)$ \\
\hline Respiratoria & 4 & $3(6,5)$ \\
\hline $\begin{array}{l}\text { Otras (cutánea, digestivo } \\
\text { portal, irugía reciente, } \\
\text { marcapasos) }\end{array}$ & $8(17,3)$ & $5(10,8)$ \\
\hline Ninguna & $24(52,1)$ & $26(56,5)$ \\
\hline
\end{tabular}

(a) diez pacientes con válvula protésica, y cuatro pacientes con marcapasos. ${ }^{\text {(b) }}$ nueve pacientes con válvula protésica, y un paciente con marcapasos. ${ }^{(c)}$ tres pacientes eran portadores de marcapasos. ${ }^{(d)}$ tres neoplasias de próstata, dos de vejiga urinaria, dos linfomas y un cáncer de colon. ${ }^{*} \mathrm{p}<0,05$

\section{HALLAZGOS ECOCARDIOGRÁFICOS}

La ecocardiografía transesofágica comenzó a realizarse en nuestro hospital en junio de 1994 y fue practicada en 29 $(62,9 \%)$ pacientes del grupo de ancianos y en $21(45,6 \%)$ casos del grupo de adultos jóvenes, como primera opción en tres pacientes con EI sobre válvula protésica y en un paciente por sospecha de extensión paravalvular de la infección, respectivamente de cada grupo. No se realizó dicha técnica en los casos que se obtuvieron hallazgos ecocardiográficos positivos y una buena calidad de imagen transtorácica. El rendimiento diagnóstico de la ecocardiografía para detectar vegetaciones, insuficiencia valvular o abscesos valvulares en los dos grupos considerados se presenta en la tabla III. La tasa de
TABLA II

DISTRIBUCIÓN DE LOS MICROORGANISMOS AISLADOS EN 92 EPISODIOS DE ENDOCARDITIS INFECCIOSA EN PACIENTES ANCIANOS Y ADULTOS JÓVENES

\begin{tabular}{|c|c|c|c|}
\hline \multirow[b]{2}{*}{ Microorganismos } & \multicolumn{3}{|c|}{$\mathrm{N}^{\circ}$ de episodios } \\
\hline & $\begin{array}{l}\text { Ancianos } \\
(n=46)\end{array}$ & $\begin{array}{l}\text { Adultos jóvenes } \\
\quad(n=46)\end{array}$ & $p$ \\
\hline \multicolumn{4}{|l|}{ Estreptococos } \\
\hline Grupo viridans & $4(8,6)$ & $11(23,8)$ & 0,04 \\
\hline Streptococcus bovis & 4 & 5 & \\
\hline Enterococcus faecalis & 3 & 3 & \\
\hline Otros & 1 & 2 & \\
\hline Total (\%) & $12(26,0)$ & $21(45,6)$ & NS \\
\hline \multicolumn{4}{|l|}{ Estafilococos } \\
\hline Staphylococcus aureus & 9 & 7 & \\
\hline $\begin{array}{c}\text { Staphylococcus } \\
\text { epidermidis }\end{array}$ & $9(19,5)$ & $3(6,5)$ & 0,06 \\
\hline Otros & 1 & 0 & \\
\hline Total (\%) & $19(41,3)$ & $10(21,7)$ & NS \\
\hline Bacilos gramnegativos & $3(6,5)$ & $1(2,1)$ & \\
\hline Otros microorganismos & $2(4,3)^{a}$ & $4(8,6)^{b}$ & \\
\hline Hongos & $2^{c}$ & $1^{\mathrm{d}}$ & \\
\hline Polimicrobiana & $2^{e}$ & $2^{f}$ & \\
\hline Hemocultivo negativo & $6(13,0)$ & $7(15,2)$ & \\
\hline
\end{tabular}

(a) Actinobacillus actinomycetemcomitans y Chlamydia sp. (b) Corynebacterium xerosis en dos casos, Neisseria mucosa y Listeria monocytógenes. (c) Candida albicans y Aspergillus fumigatus. (d) C. albicans (e) S. epidermidis y C. albicans, S.aureus y S. epidermidis. (f) E. faecalis, C. albicans, S. epidermidis y Propionibacterium acnes. NS: no significativo

TABLA III

HALLAZGOS ECOCARDIOGRÁFICOS EN 92 EPISODIOS DE ENDOCARDITIS INFECCIOSA (EI) EN ANCIANOS Y EN PACIENTES ADULTOS JÓVENES

\begin{tabular}{lcc}
\hline & \multicolumn{2}{c}{$\mathrm{N}^{\circ}$ de episodios } \\
\cline { 2 - 3 } & $\begin{array}{c}\text { Ancianos } \\
(\mathrm{n}=46)\end{array}$ & $\begin{array}{c}\text { Adultos jóvenes } \\
(\mathrm{n}=46)\end{array}$ \\
\hline $\begin{array}{l}\text { Detección de } \\
\text { vegetaciones por ETT }\end{array}$ & $20(46,5)$ & $29(64,4)$ \\
$\begin{array}{l}\text { Detección de vegetaciones } \\
\text { incluyendo ETE }\end{array}$ & $39(84,7)$ & $43(93,4)$ \\
$\begin{array}{l}\text { Diagnóstico adicional } \\
\text { alcanzado por ETE }\end{array}$ & $16(37,2)^{\mathrm{a}}$ & $13(28,8)^{\mathrm{b}}$ \\
$\begin{array}{l}\text { Insuficiencia valvular } \\
\text { Abscesos valvulares }\end{array}$ & $26(56,5)$ & $27(58,6)$ \\
& $4(8,6)^{\mathrm{c}}$ & $2(4,3)^{\mathrm{d}}$ \\
\hline
\end{tabular}

ETT: ecocardiografía transtorácica. ETE: ecocardiografia transesofágica

(a) El sobre válvula nativa en 11 casos, El sobre válvula protésica en 4 casos, y El sobre marcapasos en un caso.

(b) El sobre válvula nativa en 8 casos, El sobre prótesis valvular en 4 casos, y El sobre marcapasos en un caso. ${ }^{(c)} \mathrm{y}^{\text {(d) }}$ los abscesos paravalvulares fueron detectados por ETE. 
detección de vegetaciones por ecocardiografía transtorácica fue del $46,5 \%$ en el colectivo de ancianos, pero más alta en el grupo de enfermos jóvenes $64,4 \%$ y la ecocardiografia transesofágica incrementó la sensibilidad para detectar EI a un nivel superior al $84 \%$ en ambos grupos. La ecocardiografia transesofágica reveló un absceso paravalvular en 4 de 20 prótesis valvulares y 2 fueron observados en endocarditis sobre válvula nativa. Además, esta modalidad ecocardiográfica permitió detectar vegetaciones en dos de los cuatro casos de endocarditis sobre marcapasos.

\section{MANIFESTACIONES Y CURSO CLÍNICO}

La duración de los síntomas antes de la hospitalización fue diferente entre los dos grupos, con una media de 28 días en los pacientes mayores de 65 años, comparada con 34 días en los enfermos jóvenes. Sin embargo, la duración media de los síntomas para todos los pacientes con EI por S. aureus fue 21 días más corta que la recogida para la endocarditis debida a otras bacterias.

El análisis comparativo entre ambos grupos queda reflejado en la tabla IV. La incidencia de insuficiencia cardíaca y la tasa de mortalidad fue más elevada en los episodios de EI observados para el grupo de ancianos, aunque no se encontraron diferencias significativas en estas variables o en la presencia de fiebre, signos periféricos, manifestaciones musculoes-

\section{TABLA IV}

MANIFESTACIONES CLÍNICAS Y EVOLUCIÓN EN 92 EPISODIOS DE ENDOCARDITIS INFECCIOSA EN PACIENTES ANCIANOS Y EN ADULTOS JÓVENES

\begin{tabular}{lcc}
\hline & \multicolumn{2}{c}{$\mathrm{N}^{\circ}$ de episodios } \\
\cline { 2 - 3 } & $\begin{array}{c}\text { Ancianos } \\
(\mathrm{n}=46)\end{array}$ & $\begin{array}{c}\text { Adultos jóvenes } \\
(\mathrm{n}=46)\end{array}$ \\
\hline Fiebre $\left(>38^{\circ} \mathrm{C}\right)$ & $39(84,7)$ & $44(95,6)$ \\
Fenómenos microvasculares & $2(4,3)$ & $6(13)$ \\
periféricos & & \\
Esplenomegalia & $8(17,3)$ & $12(26)$ \\
Insuficiencia cardiaca & $18(39,1)^{\mathrm{a}}$ & $10(21,7)$ \\
Embolismos & $10(21,7)$ & $9(19,5)$ \\
$\quad$ cerebral & 9 & $5^{\mathrm{c}}$ \\
pulmonar & 1 & 0 \\
visceral & 0 & 3 \\
periférico & 0 & 1 \\
Alteraciones renales & $2(4,3)$ & $6(13)$ \\
insuficiencia renal & $8(17,3)^{*}$ & $2(4,3)$ \\
microhematuria & $8(17,3)$ & $15(32,6)$ \\
Manifestaciones & $11(23,9)^{\mathrm{d}}$ & $12(26)^{\mathrm{e}}$ \\
musculoesqueléticas & $17(36,9)$ & $18(39,1)$ \\
Cirugía cardiaca & $9(19,5)$ & $5(10,8)$ \\
$\quad$ Mortalidad & &
\end{tabular}

(a) Fallecieron 8 pacientes, cinco de ellos fueron rechazados para cirugía. A 8 se le realizó cirugía valvular, sólo uno falleció. (b) Ictus isquémico en 7 casos, e ictus hemorrágico en 2 casos. ${ }^{(c)}$ Ictus isquémico en 3 casos, aneurisma micótico en un caso y absceso cerebral en otro caso.

(d) Artromialgias en 5 casos, espondilitis lumbar en 5 casos, y artritis de muñeca en un caso. ${ }^{(e)}$ Artromialgias en 10 casos, artritis de tobillo en dos casos

* $p<0,05$ comparado con el grupo de adultos jóvenes. queléticas, embolismos, esplenomegalia o necesidad de cirugía. La insuficiencia renal al ingreso $(\mathrm{p}=0,04)$ fue la única variable que resultó significativamente más común entre la población anciana.

Se realizó tratamiento quirúrgico en fase activa en 35 de los 92 episodios de EI, siendo el porcentaje de pacientes operados similar entre los dos grupos (Tabla IV). Las indicaciones de cirugía en el colectivo de pacientes de mayor edad fueron insuficiencia cardíaca en ocho casos, bacteriemia persistente en cuatro casos, absceso paravalvular en tres y episódios embólicos recurrentes en dos casos. En los enfermos más jóvenes 12 de ellos fueron operados por insuficiencia cardíaca congestiva; y dos casos por fiebre persistente, embolismos, y abscesos, respectivamente. Los cuatro pacientes con endocarditis sobre marcapasos presentaron una bacteriemia continua por S. epidermidis y fue necesaria la extracción bajo circulación extracorpórea de todo el sistema (generador y electrodos). Nueve pacientes del grupo de ancianos (19,5\%) y cinco adultos jóvenes (10,8\%) fallecieron durante la hospitalización. La insuficiencia cardíaca fue la principal causa de muerte en los dos grupos, $8 / 9(88,8 \%)$ de los ancianos y 2/5 (40\%) pacientes del grupo de menor edad. Cabe señalar que cinco de los enfermos mayores de 65 años fueron rechazados para cirugía valvular por comorbilidad grave (cuatro pacientes con cáncer y un paciente con un infarto de miocardio reciente), fallecieron.

\section{DISCUSIÓN}

Tradicionalmente se ha considerado que la edad avanzada se asocia con manifestaciones atípicas y un peor pronóstico de la EI $(13,14)$. Sin embargo, la mayoría de los estudios han sido realizados antes de la década de los noventa (15-17), cuando aún no se disponía de unos criterios diagnósticos uniformes y sensibles para algunos tipos de endocarditis, ni de nuevas técnicas de diagnóstico ecocardiográfico y microbiológico, y no tienen en cuenta los últimos cambios ocurridos en la epidemiología de la enfermedad (1). En contraste con esta percepción general, los resultados de nuestro estudio sugieren que las características clínicas y la evolución de la EI en nuestro medio son similares para los ancianos y los pacientes adultos jóvenes. Estos datos coinciden globalmente con los aportados más recientemente por otros autores $(9-11,18,19)$, que también utilizan los criterios de Duke para la selección de pacientes con endocarditis.

La edad de los pacientes que desarrollan una EI ha aumentado notablemente en las últimas décadas. Así, en estudios recientes $(2,3,11)$ la edad media se sitúa entre los 55 y 67 años, comparable a la de nuestra serie que fue de 60 años, y con una alta proporción de varones, a diferencia de otros estudios que encuentran una proporción similar entre ambos sexos (17) e incluso superior para las mujeres (1).

La distribución de la enfermedad cardíaca subyacente presentó una alta incidencia de endocarditis sobre material protésico en los ancianos $(30,4 \%)$. Este hecho podría estar en relación con la mayor prevalencia de válvulas protésicas y de marcapasos en este grupo de población, quienes más a menudo necesitan de cuidados médicos y, en consecuencia, es más probable que sean sometidos a procedimientos invasívos bacteriémicos. Los ancianos con dispositivos protésicos intracardiacos se considera que presentan un alto riesgo de EI $(7,9)$. En nuestra serie, un tercio de los de los enfermos de cada gru- 
po no presentaba enfermedad cardiaca predisponente. Cabe señalar, por tanto, que la ausencia de una cardiopatía previa conocida no descarta el diagnóstico de EI. La localización topográfica de la infección fue similar en ambos grupos. Otras series obtienen resultados variables, con predominio de la afectación mitral (7) o aórtica (18) en los ancianos. Como cabía esperar, las enfermedades de base y comorbilidad fueron más prevalentes en el grupo de mayor edad, que presentaron una incidencia de cáncer significativamente alta. Estos hallazgos han sido comunicados por otros autores $(19,20)$, que también encuentran una neoplasia subyacente como el factor predisponente más común para el desarrollo de una EI en pacientes ancianos.

No hallamos diferencias significativas en la distribución global de los microorganismos causales entre los dos grupos de edad. Sin embargo, en nuestro estudio encontramos un alto porcentaje de EI debida a estafilococos (41\%) en los ancianos con respecto a los descrito en otras series $(9,18)$, donde predominan los estreptococos, fundamentalmente enterococos y $S$. bovis, o se distribuyen a partes iguales entre ambos microorganismos (7), en relación con la mayor frecuencia en este colectivo de una puerta de entrada y fuente de bacteriémia urinaria o digestiva portal, que fue poco común en nuestros pacientes.

Los ancianos tienen una mayor incidencia de lesiones valvulares degenerativas, de válvulas protésicas y de marcapasos que reducen de forma notable la sensibilidad de la ecocardiografía transtorácica para detectar vegetaciones o abscesos $(21,23,24)$. En la presente serie, el incremento de la sensibilidad ecocardiográfica de la vía transesofágica resultó especialmente útil en estos pacientes, lo que evitó retrasos en el inicio de un tratamiento apropiado, como ha sido referido previamente (9).

Desde el punto de vista clínico encontramos signos y síntomas de EI similares en ambos grupos. Especialmente, la presencia de fiebre, que ha sido descrita ocurre con menor frecuencia en los pacientes ancianos $(7,25)$. Aunque existió una tendencia a presentar un aumento de la incidencia de insuficiencia cardiaca, embolismo cerebral y mortalidad durante los episodios que fueron observados para los ancianos no fue significativa la diferencia en estas complicaciones entre los dos grupos de edad. Solamente, la insuficiencia renal fue significativamente mayor entre los parientes ancianos, al igual que en otros estudios $(10,19)$.

En la presente serie, la mortalidad hospitalaria en los ancianos fue del $19,5 \%$, cifra muy inferior a la de otros trabajos previos $(7,20,26)$, que llega a ser del $45-50 \%$, pero que está en línea o incluso mejora el pronóstico de otras series más recientes $(9,13,18)$ con una mortalidad en este grupo de población que se sitúa alrededor del $25 \%$, y comparable a la observada en los enfermos más jóvenes. Estas variaciones en los porcentajes de mortalidad podrían atribuirse, al menos en parte, a diferencias en el manejo clínico y la prescripción de tratamientos menos agresivos en los pacientes de mayor edad. En este sentido, la utilización restrictiva de la cirugía es probablemente la responsable del peor pronóstico observado en pacientes ancianos $(16,18)$. Sin embargo, esto no impide que precisamente en este grupo de edad haya que ser muy estrictos en la valoración de las enfermedades y factores de comorbilidad, y es obvio que las contraindicaciones a la cirugía son más prevalentes en los ancianos. En nuestro estudio, los pacientes mayores de 65 años recibieron un tratamiento similar al de los más jóvenes incluyendo una elevada tasa de cirugía que fue comparable para los dos grupos en casos de insuficiencia cardiaca como consecuencia de la destrucción valvular, bacteriemia persistente, embolismos recurrentes, abscesos paravalvulares y endocarditis sobre marcapasos. Nuestros resultados confirman las observaciones de otros estudios (9-11), que han destacado la necesidad de prescribir tratamientos más agresivos a los pacientes ancianos con EI para mejorar su evolución global.

Este trabajo presenta las limitaciones habituales de series retrospectivas que han sido recogidas durante un largo período de tiempo y que, como en la mayoría de los estudios publicados, nuestra serie se ha realizado en un hospital terciario lo que puede haber impactado en la presentación clínica, tasa de cirugía y evolución de la EI, como indican otros autores $(9,10)$. Sin embargo, cabe mencionar que solamente un pequeño número de pacientes fue trasladado a nuestro centro desde otros hospitales. Por otra parte, aunque no disponemos de información del seguimiento de los pacientes afectados de EI, otros estudios $(9,11)$ sugieren que los pacientes de edad avanzada tienen una supervivencia a corto y largo plazo comparable a la de otros grupos de población más jóvenes.

En definitiva, hemos encontrado que los pacientes ancianos representan una notable proporción de los casos de EI, y se han objetivado escasas diferencias clínicas o bacteriológicas con el resto de la población, así como en el pronóstico de la enfermedad. Sin embargo, los determinantes de la evolución son complejos y nuestros datos sugieren que, al ser considerada de forma aislada, la edad resulta insuficiente para definir el riesgo y las estrategias terapéuticas. Por consiguiente, la edad no debería utilizarse como criterio fundamental para denegar un tratamiento temprano y enérgico a los pacientes con EI.

\section{Bibliografía}

1. Hogevik H, Olaison L, Anderson R, Lindberg J. Epidemiologic aspects of infective endocarditis in an urban population. A 5-year prospective study. Medicine (Baltimore) 1995; 74: 324-339.

2. Van der Meer JTM, Thompson J, Valkemburg HA, Michel MF. Epidemiology of bacterial endocarditis in the Netherlands. I. Patients characteristics. Arch Intern Med 1992; 152: 1863-1868.

3. Delahaye F, Goulet V, Lacassin F, Ecochard R, Selton-Suty C, Hoen B, et al. Characteristics of infective endocarditis in France in 1991: a one year survey. Eur Heart J 1995; 16: 394-401.

4. Censo de Población Español. Instituto Nacional de Estadística, 2001.

5. Steckelberg JM, Murphy JG, Ballard D, Bailey K, Takij AJ, Taliencis $\mathrm{CP}$, et al. Emboli in infective endocarditis: the prognostic value of the echocardiography. Ann Intern Med 1991; 114: 635-640.

6. Ribera Casado JM. Endocarditis infecciosa en el anciano. Rev Esp Cardiol 1998; 51(Supl 2): 64-70.

7. Terpenning MS, Buggy BP, Kauffman CA. Infective endocarditis: clinical features in young and elderly patients. Am J Med 1987; 83: 626634. 
8. Thell R, Martin FH, Edwards JE. Bacterial endocarditis in subjects 60 years of age and older. Circulation 1975; 51: 174-182.

9. Werner GS, Schulz R, Fuchs JB, Andreas S, Prange H, Ruschewski W, et al. Infective endocarditis in the elderly in the era of transesophageal echocardiography: clinical features and prognosis compared with younger patients. Am J Med 1996; 100: 90-97.

10. Gagliardi JP, Nettles RE, McCarty DE, Sanders LL, Corey GR, Sexton DJ. Native valve infective endocarditis in elderly and younger adult patients: comparison of clinical features and outcomes with use of the Duke criteria and the Duke endocarditis database. Clin Infect Dis 1998; 26: $1165-1168$

11. Paccalin M, Pasdeloup T, Cazenave-Roblot F, Ragot S, Breux JP, RaudRaynier P, et al. Influence de l’âge sur le pronostic de l'endocardite infectieuse. Arch Mal Coeur 2000; 93: 1083-1087.

12. Durack DT, Lukes AS, Bright DK, and the Duke Endocarditis Service. New criteria for diagnosis of infective endocarditis: utilization of specific echocardiographic findings. Am J Med 1994; 96: 200-209.

13. Gleckler WJ. Diagnostic aspects of subacute bacterial endocarditis in the elderly. Arch Intern Med 1959; 102: 761-765.

14. Korzeniowski OM, Kaye D. Infective endocarditis. In: Braunwald EG, ed. Heart Disease, Philadelphia. Saunders; 1992: 1078-1105.

15. Cantrell M, Joshikawa TT. Infective endocarditis in the aging patient. Gerontology 1984; 30: 316-326.

16. Applefeld MM, Homick RB. Infective endocarditis in patients over age 60. Am Heart J 1974; 88: 90-94.

17. Watanakunakorn $\mathrm{C}$, Burkert $\mathrm{T}$. Infective endocarditis at large community teaching hospital, 1980-1990. A review of 210 episodes. Medicine (Baltimore) 1993; 72: 90-102.
18. Selton-Suty C, Hoen B, Gretzinger A, Houplon P, Maignan M, Juillière $\mathrm{Y}$, et al. Clinical and bacteriological characteristics of infective endocarditis in the elderly. Heart 1997: 77: 260-263.

19. Netzer RO, Zollinger E, Seiler C, Cerny A. Native valve infective endocarditis in elderly and younger adult patients: comparison of clinical features and outcomes with use of the Duke criteria. Clin Infect Dis 1999; 28: 933-934.

20. Domínguez Arganda L, Vázquez Pedrazuela, García Navarro JA, Sánchez-Harguindey, Ribera Casado JM. Endocarditis infecciosa en el anciano: estudio retrospectivo entre 1990-1994. Rev Esp Geriatr Gerontol 1995; 30: 301-307.

21. Birmingham GD, Rahko PS, Ballantyme III. Improved detection of infective endocarditis with transesophageal echocardiography. Am Heart J 1992; 123: 774-781.

22. Daniel WG, Mugge A, Martin RP, Lindert O, Hausmann D, NonnastDaniel B, et al. Improvement in the diagnosis of abscess associated with endocarditis by transesophageal echocardiography. N Engl J Med 1991; 324: 795-800.

23. Villacosta I, Sarriá C, San Román JA, Jimenez J, Castillo JA, Iturralde E, et al. Usefulness of transesophageal echocardiography for diagnosis of infected transvenous permanent pacemakers. Circulation 1994; 89: 2684-2687.

24. Dhawan VK. Infective endocarditis in elderly patients. Clin Infect Dis 2002; 34: 806-812.

25. Finkelstein MS. Unusual features of infections in the aging. Geriatric 1982; 37: 65-78.

26. Castillo JC, Anguíta MP, Ramírez A, Siles J, Mesa D, Muñoz I et al Características clínicas y pronósticas de la endocarditis infecciosa en el anciano. Rev Esp Cardiol 2000; 53:1437-1442. 